\title{
BedRest Alters ResPiratory Muscle STRENGTH In PATIENTS IMMOBILIZED Due to Fractured FeMURS
}

\begin{abstract}
Bedrest produces decrements in the functioning of all physiological systems. Physiotherapists treat patients who are managed with Thomas splints following fractured femurs. The patients are generally compartmentally treated as orthopaedic patients. No attention is paid to the status of the respiratory system which can be the cause of morbidity in these patients. Since the ventilatory muscles play a major role in breathing and coughing the purpose of

\section{PUCKREE T, BSC, MS, PhD; MOONASUR R, B Physio,; GOVENDER K, B Physio}

\footnotetext{
${ }_{2}^{1}$ Department of Physiotherapy, University of Durban-Westville.

2 Basic Physiotherapist, King Edward VIII Hospital, Durban.

3 Basic Physiotherapist, Westville Hospital, Durban.
} the present study was to determine the effect of a limited period of bedrest on the strength of the inspiratory and expiratory muscles.

A sample of convenience consisting of 15 African male patients immobilized by Thomas' Splint for fractured mid-shaft femurs participated in the study by voluntary consent. All patients had been confined to bed for at least 3 weeks. Maximal inspiratory pressures (PiMAX) and Maximal expiratory pressures (PeMAX)were recorded using a Boehringer Force Meter at functional residual capacity in the semi-recumbent position. The data were normalized and analyzed using the student t-test at the $0.05 \%$ level.

The results showed a significant reduction in both PiMAX and PeMAX compared to age predicted normal values. The decrease in PeMAX was greater than that of PiMAX.

We conclude that respiratory muscle strength decays with bedrest.
\end{abstract}

\section{KEYWORDS: KEY WORDS : BEDREST, RESPIRATORY MUSCLES, WEAKNESS}

\section{This research was presented as a poster at the 13 th international conference of the World Confederation of Physical Therapy held in Yokohama in May, 1999.}

\section{INTRODUCTION :}

Following certain types of injury, otherwise healthy subjects are surgically or medically managed in a supine or semirecumbent position for three to twelve weeks. The orthopaedic management of certain categories of fractured femurs includes skeletal traction using a Thomas' splint. Fractures of the lower limb take a average 12 weeks to unite hence patients are kept in traction for more than 3 weeks. However, bedrest results in the deconditioning of several physiological systems (Brooks and Fahey, 1984) resulting in impairments, disability and handicap even in young patients. Asher's (1947) request to get patients out of bed and save them an early grave, is sufficient to imply the detrimental effects of bedrest on the normal functioning of the various systems of the body.

The respiratory system plays an important role in providing the body with oxygen and in the removal of carbon-dioxide ( $\mathrm{CO} 2)$. The effective functioning of the respiratory system is partially dependent on the strength of the respiratory muscles which essentially act as a pump. The function of the respiratory muscles can be directly affected by bedrest and indirectly affected by the position in which bedrest occurs ( $\mathrm{Ng}$ and Stokes, 1991). The type and duration of bedrest can reduce maximal oxygen consumption (V02max) and work capacity from as little as $1 \%$ to about $26 \%$ (Brooks and Fahey, 1984). In supine, the vital capacity falls by $8-10 \%$ and with diaphragm weakness it may fall by more than 30\% (Campbell et. al, 1970).

The strength of the respiratory muscles determines the amount of inspiratory and expiratory pressures that can be developed (Clanton and Diaz, 1989). The latter affects pulmonary volumes and timing, hence breathing depth and frequency. Bedrest can produce decrements in the force production or strength and endurance of the respiratory muscles (Clanton and Diaz, 1989, Chase et.al, 1966). This reduction in respiratory muscle strength can result in poor ventilation of the lungs. Poorly ventilated areas can be susceptible to infection.

No studies have investigated the effects of bedrest on respiratory muscle strength in orthopaedic patients. The strength of the respiratory muscles can be assessed clinically by measuring the

\section{CORRESPONDENCE:}

T. Puckree

Senior lecturer

Department of Physiotherapy, University of Durban-Westville, Private Bag X54001 Durban, 4000, South Africa

Tel: (031) 204-4977

Fax: (031) 204-4817

Email: lpuckree@pixie.udw.ac.za 
pressure generated by these muscles (Clanton and Diaz, 1989). The maximum inspiratory pressure (PiMax) and maximum expiratory pressure (PeMax) are indices of global inspiratory and expiratory muscle strength respectively. These measures are considered to be sensitive measures of developing muscle weakness. Although not suitable for patients with rapidly developing muscle weakness they are ideal for use on orthopaedic patients (Clanton and Diaz, 1989). Each of these parameters is easy to measure using a calibrated manometer or electronic gauge (Clanton and Diaz, 1989).

The purpose of the present study was to determine whether patients with fractured femurs on bedrest of more than 3 weeks duration develop weakness of the respiratory muscles.

\section{METHODS:}

The sample of convenience consisted of 15 black males with ages ranging from 20 to 45 years. All patients had sustained femoral midshaft fractures and were managed on skeletal traction. The patients were included if they had no history of smoking or an upper respiratory tract infection in the 2 weeks prior to the study. These patients also had no history of cardiopulmonary or neuromuscular disease. All patients included in the study gave their full informed consent (by signing a consent form) to participate in the study which had ethical approval from the Ethics Committee of the University of Durban-Westville.

\section{INSTRUMENTATION}

A calibrated inspiratory/expiratory force meter (Boehringer Laboratories, Gounden,1985) with a built in leak previously used on quadruplegic patients was used to record PiMax and PeMax. A standard plastic noseclip used in physiology laboratories was used to seal the nose during breathing.

\section{PROCEDUIRE}

Every patient who met the inclusion criteria at one state hospital and had been on bedrest for a duration of 3 weeks or more between May and August of 1997, was included in the study. All the data were collected by the same investigator to eliminate inter-rater errors Each patient was monitored in the semi-recumbent position. The procedure to be followed (as described below) was explained to each subject and the process demonstrated. The mouth was sealed around a standard spirometer cardboard mouth-piece attached to the force meter. Because PiMax recordings were taken at functional residual capacity (FRC), each subject was asked to relax completely, then to inspire rapidly and maximally and hold the breath for one second. A leak on the force meter prevented the patient from using the buccal muscles to generate pressure. The procedure was repeated five times with a one minute rest between the readings. For the recording of PeMax, the subject was asked to relax completely and then breathe out rapidly and completely. The highest of each set of five readings was used in the statistical analysis.

\section{DATA ANALYSIS}

To minimize variation between subjects and reduce total error each subject's measured value had to be normalized in relation to that subject' age predicted value.

Age predicted normal values for each subject was calculated using the following formulae (Clanton and Diaz, 1989).

$\mathrm{Pi}_{\text {Max }}$ (adult male) in $\mathrm{kPa}$

20-65 years (not race adjusted) $=$

$-14.02+0,054 *$ age

measurement at FRC =

value obtained decreased by $14 \%$

converted to $\mathrm{cm} \mathrm{H} 20=\left(\mathrm{kPa}^{*} 10.2\right)$

$\mathrm{Pe}_{\text {Max }}$ (adult male) in $\mathrm{kPa}$

20-65 years (not race adjusted) $=$

$26,27+0,101 *$ age

measurement at $\mathrm{FRC}=$ value

obtained decreased by $19 \%$

converted to $\mathrm{cm} \mathrm{H} 20=(\mathrm{kPa} * 10.2)$

Each subject's measured value was normalized to that individuals age predicted value by calculating the measured value as a percentage of the age predicted value. Means and standard deviations were calculated. Normalized values were compared to age predicted values by using a two tailed student t-test with a probability set at 0,05 .

\section{RESULTS :}

Table 1 shows the measured, age predicted normal, and normalized values for each of the 15 subjects. As shown only 2 of the 15 patients retained at least $58 \%$ of their predicted $\mathrm{Pi}_{\mathrm{Max}}$ and only one patient was capable of producing at least $30 \%$ of predicted Pe $_{\text {Max }}$. However the latter patient had the lowest $\mathrm{Pi}_{\mathrm{Max}}$ value of $28 \%$.

Figure 1 shows bar graphs of the measured and age predicted values of $\mathrm{Pi}_{\mathrm{Max}}$ and $\mathrm{Pe}_{\mathrm{Max}}$. A significant decrease in both measured $\mathrm{Pi}_{\mathrm{Max}}$ and $\mathrm{Pe}_{\mathrm{Max}}$ was observed compared to age predicted normal values. In addition a significantly greater decrease was observed in $\mathrm{Pe}_{\mathrm{Max}}$ values compared to $\mathrm{Pi}_{\mathrm{Max}}$ values.

\section{DISCUSSION:}

The strength of respiratory muscles determines the amount of inspiratory and expiratory pressures that can be developed during inspiration and expiration (Clanton and Diaz, 1989). The latter affects pulmonary volumes and timing, hence breathing depth and frequency. The results obtained demonstrate that both inspiratory and expiratory muscle strength was decreased due to bedrest in the study sample. The findings are consistent with reports in the literature about decrements in various pulmonary parameters like $\mathrm{VO}_{2}$ max, vital capacity, etc with bedrest ( Brooks and Fahey, 1984).

Clanton and Diaz(1989) reported that $\mathrm{Pi}_{\text {Max }}$ and $\mathrm{Pe}_{\text {Max }}$ values are dependent on lung volumes. Resting lung volumes affect the length of the muscles which influences the ability of each muscle to generate force (Campbell et al., 1970, De Troyer, 1983). The diaphragm is at an optimal position for generating force in the supine position (Campbell et al,1970). If the patients were on bedrest in the semi-recumbent position as in this study, it is likely that the diaphragm was not only operating at a less than optimal length, but also was not exerted to its full potential at each breath, gradually resulting in decrements in respiratory 
TABLE 1. Age, predicted, the measured $\mathrm{Pi}_{\text {Max }}$ and Pe $\mathrm{Max}_{\text {ax }}$ and normalized values $(\%)$ for each of the 15 subjects

\begin{tabular}{|c|c|c|c|c|c|c|c|}
\hline Subject & Age & $\begin{array}{c}\text { Predicted } \\
\text { PiMax }\end{array}$ & $\begin{array}{c}\text { Measured } \\
\text { PiMax }\end{array}$ & $\begin{array}{c}\text { Normalized } \\
\text { values }\end{array}$ & $\begin{array}{c}\text { Predicted } \\
\text { PeMax }\end{array}$ & $\begin{array}{c}\text { Measured } \\
\text { PeMax }\end{array}$ & $\begin{array}{c}\text { Normalized } \\
\text { Values }\end{array}$ \\
\hline 1 & 26 & 135.3 & 65.6 & 48.5 & 195.4 & 45.7 & 23.4 \\
\hline 2 & 34 & 139.1 & 62.4 & 44.9 & 188.7 & 46.0 & 24.4 \\
\hline 3 & 36 & 140 & 61.2 & 43.7 & 187.0 & 46.0 & 24.6 \\
\hline 4 & 25 & 134.8 & 71.5 & 53.0 & 196.2 & 50.7 & 25.8 \\
\hline 5 & 34 & 139.1 & 63.9 & 45.9 & 188.7 & 39.7 & 21.0 \\
\hline 6 & 41 & 142.4 & 63.0 & 44.2 & 182.8 & 50.1 & 27.4 \\
\hline 7 & 24 & 134.4 & 64.0 & 47.6 & 197.0 & 42.9 & 21.8 \\
\hline 8 & 28 & 136.3 & 38.6 & 28.3 & 193.7 & 60.9 & 31.4 \\
\hline 9 & 25 & 134.8 & 78.8 & 58.5 & 196.2 & 43.4 & 22.1 \\
\hline 10 & 29 & 136.7 & 74.1 & 54.2 & 192.8 & 49.2 & 25.5 \\
\hline 11 & 35 & 139.6 & 59.9 & 42.9 & 187.8 & 50.8 & 27.0 \\
\hline 12 & 38 & 141.0 & 63.6 & 45.1 & 185.3 & 52.8 & 28.5 \\
\hline 13 & 36 & 140.0 & 82.1 & 58.6 & 187.0 & 54.5 & 29.1 \\
\hline 14 & 42 & 142.9 & 63.5 & 44.4 & 182.0 & 37.6 & 20.7 \\
\hline 15 & 29 & 136.7 & 68.5 & 50.1 & 192.8 & 52.7 & 27.3 \\
\hline Mean & 32 & 135.3 & 65.4 & 48.3 & 190.3 & 48.2 & 25.3 \\
\hline
\end{tabular}

FIGURE 1:

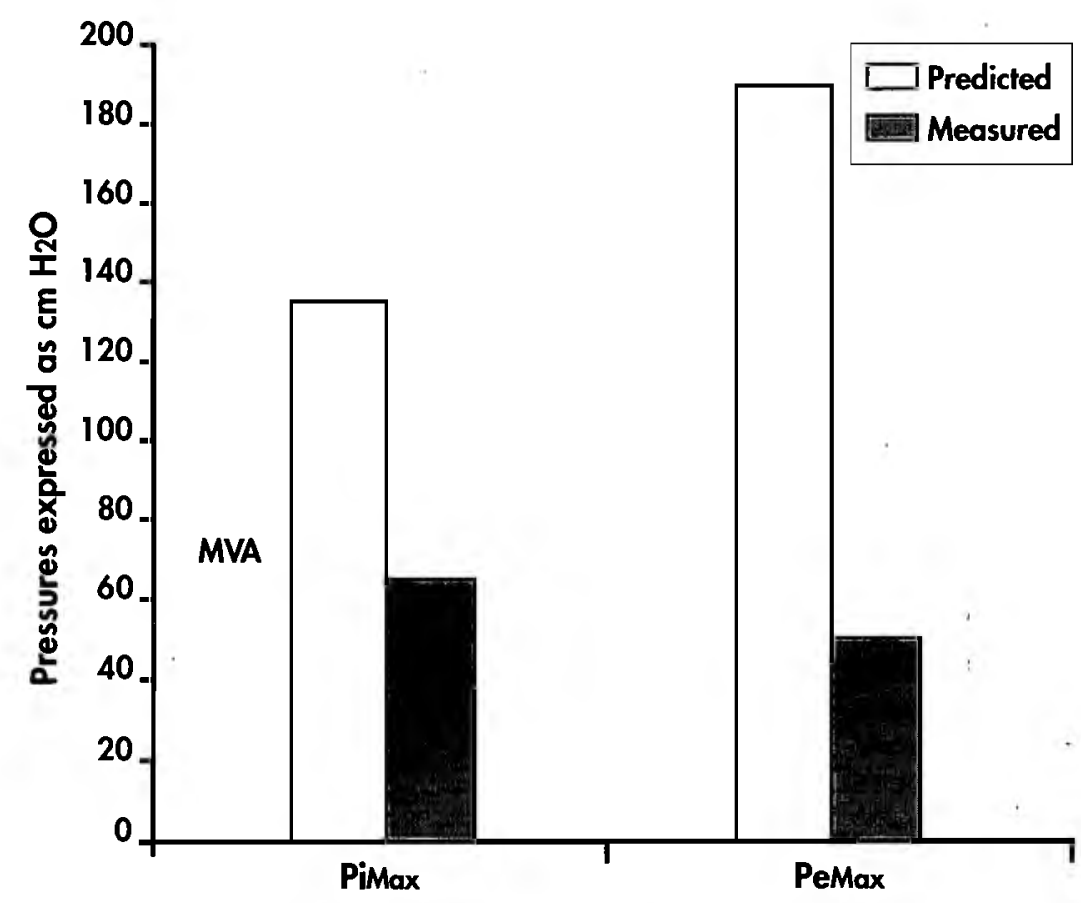

Pressures as Indices of Inspiratory and Expiratory Muscle Strength

muscle strength. Similar to other skeletal muscles that are immobilized in prescribed positions, the diaphragm and the other respiratory muscles could develop contactures. The latter affects the strength producing ability of these muscles in the short and long terms if sustained.

It has also been shown that the type and duration of bedrest can reduce $\mathrm{VO}_{2} \mathrm{max}$, and work capacity from as little as $1 \%$ to about $26 \%$ (Brooks and Fahey, 1984). In these subjects 3 weeks of bedrest in the semi-recumbent position resulted in a loss of up to $50 \%$ of inspiratory muscle strength and $75 \%$ of expiratory muscle strength. Large decrements in respiratory muscle strength in patients similar to the study cohort can be prevented.

More dynamic approaches to the treatment of patients with femoral fractures (Brooks and Fahey,1984, Campbell et al, 1970) are recommended.

A greater decrease in expiratory muscle strength compared to inspiratory 
muscle during the duration of bedrest was noted. Since both the inspiratory and expiratory muscles are skeletal muscles, one would expect a decrement in muscle strength as shown in this study. The sample consisted of young male patients - this could have affected the results in one direction or another. Young patients usually come from the active labour force and therefore should have healthy cardio-pulmonary endurance which requires healthy respiratory muscles. Muscles that are well developed or are of the fast fatigable type, lose strength rapidly due to disuse. It is known that both the inspiratory and expiratory muscles consist of almost equal proportions of type I and type II fibres (Eberstein and Goodgold, 1968). If the Type II fibres loose strength rapidly and the type I fibres slowly due to limited disuse then one will find a loss of respiratory muscle strength. Since breathing is a regular event that does not stop even with bedrest, the inspiratory muscles are used but probably not to the extent that they were exerted to prior to bedrest. Since expiration is a passive process at rest and the major muscle of active expiration (the abdominals) is a multifunctional muscle, one would expect the other functional components of the muscle, like postural control, to lose strength rapidly. On the other hand it has been shown that different task groups within the abdominal motor pools control different tasks (Puckree et.al, 1998). Other groups of neurons are multifunctional. Is it possible that the greater loss of expiratory muscle strength is due to the loss of strength in motor units that become active during more than one task?

In conclusion the results of this study clearly indicate decrements in respiratory muscle strength due to bedrest. More studies on a larger sample size, different age groups, gender and population groups will yield more information which may be used to inform physiotherapy practice in this clinical area. In addition, the study parameters must be recorded on admission, and at one monthly intervals until the patient is allowed out of bed.

\section{REFERENCES:}

Asher RAJ 1947 The dangers of going to bed. British Medical Journal 4: 967 -968

Brooks AJ, Fahey D 1984 Exercise Physiology: Human bioenergetics and its applications. Macmillan Publishing Company, New York: 4
Campbell EJM, Agostone E, Davis JN 1970 Ed. The respiratory muscles: mechanics and control.W.B. Saunders company, Philadelphia: 35

Chase GA, Grave C, Rowell LB 1966 Independence of changes in functional and performance capacities attending prolonged bed rest. Aerospace Medicine 37: 1232-1238

Clanton TL and Dias PT 1989 Clinical Assessment of the respiratory muscles. Physical Therapy 75:983-995

De Troyer A 1983 Mechanical role of the abdominal muscles in relation to posture. Respiratory Physiology 53:341-3531

Eberstein A, Goodgold J 1968 Slow and fast twitch fibers in human skeletal muscle. American Journal of Physiology 215:535-54 I

Gounden P 1985 Assessment of ventilatory muscle strength using a pressure manometer. Physiotherapy 41: 79-80

Ng GY and Stokes MJ 1991 Maximal inspiratory and expiratort mouth pressures in sitting and half-lying positions in normal subjects. Respiratory Medicine 85: 209-211

Puckree T, Cerny F, Bishop B 1998 Abdominal motor unit activity during respiratory and non-respiratory tasks. Journal of Applied Physiology 84(5): 1705-1715 